\title{
Evaluación de una Mezcla Empanizadora, con Inclusión de Almidón Modificado, para su Aplicación en Carnes
}

\author{
Bautista, Norma1 iD ; Franco-Crespo, Christian ${ }^{1, ~ * ~ i D ~}$ \\ ${ }^{1}$ Universidad Técnica de Ambato, Campus Huachi, Ambato, Ecuador
}

\begin{abstract}
Resumen: La industria de los alimentos se está enfocando en agregar ingredientes alternativos que puedan mejorar las características del producto, así como también cuidar la salud de los consumidores. La investigación tiene como objetivo evaluar una mezcla de empanizado con inclusión de almidón modificado de maíz para la aplicación en carnes de pollo, pescado y res. Tres tratamientos son formulados con variación de almidón modificado (10\%, 20\% y 30\%). La metodología aplicada en este estudio considera un análisis sensorial, propiedades de color y textura, así como también la determinación del porcentaje de absorción de aceite. La mezcla con almidón modificado al 30\% presenta los mejores resultados, dando como resultado menos pérdidas durante el proceso de fritura, mediante la evaluación de los atributos sensoriales del producto empanizado. La evaluación de las propiedades de color evidencia que la inclusión de almidón modificado no afecta grandemente en sus valores, generando así una coloración dorada en un rango de $\mathrm{H}^{\circ}$ de $66,46 \pm 3,11$ a 59,65 $\pm 1,83$; mientras que en la evaluación de las propiedades de textura, se produce una mejora en la dureza, cohesividad y masticabilidad. Finalmente, la cantidad de absorción de aceite en muestras con harina comercial (MC) presenta mayor absorción de aceite con un 14,83\%, 17,72\% y 22,54\% para empanizados con carne de pollo, pescado y res respectivamente, mientras que el T3 con 30\% de almidón modificado reduce la absorción de aceite con un valor de $11,01 \%$ para pollo, 15,32\% en pescado y 16,16\% en res empanizados.
\end{abstract}

Palabras clave: Absorción de aceite, Evaluación sensorial, Propiedades de color y textura.

\section{Evaluation of a Breading Mixture, Including Modified Starch, for Application to Meat}

\begin{abstract}
Food industry is focusing on adding alternative ingredients that can improve product characteristics as well as care for the health of consumers. The research proposes to evaluate a breading mixture including modified corn starch for application in chicken, fish and beef meats. Three treatments are formulated with modified starch variation $(10 \%, 20 \%$ and $30 \%)$. The methodology applied in this study considers a sensory analysis, color and texture properties, as well as the determination of oil absorption percentage. The mixture with $30 \%$ modified starch presents the highest score in the samples, resulting in less losses during the frying process, during the sensory evaluation attributes of the breaded product. The evaluation of the color properties shows that the inclusion of modified starch does not greatly affect its values, this generates a golden coloration in an $\mathrm{H}^{\circ}$ range of $66.46 \pm 3.11$ to $59.65 \pm 1.83$; while in the evaluation of texture properties, there is an improvement in hardness, cohesiveness and chewiness. Finally, the quantity of oil absorption in samples with commercial flour (MC) presents a greater oil absorption with $14.83 \%, 17.72 \%$ and $22.54 \%$ for breaded chicken, fish and beef respectively, while T3 with $30 \%$ modified starch with a value of $11.01 \%$ for chicken, $15.32 \%$ for fish and $16.16 \%$ breaded beef.
\end{abstract}

Keywords: Oil absorption, Sensory evaluation, Color and texture properties.

\section{INTRODUCCIÓN}

Los empanizadores son denominados productos de revestimiento que se aplican a productos que van a ser sometidos a fritura (Sahin \& Gülün, 2009). Generalmente, este proceso de fritura se aplica por inmersión; siendo uno de los métodos de cocción que tiene mayor aceptabilidad, por el sabor y textura que tiene gran demanda por la sensación en el paladar de los consumidores. Sin embargo, este proceso tiene como consecuencia riesgos en la salud por la cantidad de aceite absorbido durante el proceso de fritura, que puede variar en función de la estructura que se forma en el revestimiento (Montes, Millar, y Provoste, 2016).

Los empanizados son elaborados a base de harina de trigo, arroz y maíz, a los que pueden añadirse condimentos u otras inclusiones para proporcionar textura, sabor, color y apariencia luego de la fritura (Dávalos, 2016). La aplicación 
de los empanizados es amplia y se relaciona con la elaboración de alimentos procesados, listos para consumir, mejorando su aspecto, incrementado volumen del producto y generando una cobertura que brinda cualidades sensoriales, que potencia el sabor de diversas carnes. Por este motivo, la aplicación de empanizados es amplia en la industria de alimentos. Además, puede contemplar varios tipos de cobertura, como son: rebozados, empanizados en productos sometidos a preparación completa o incompleta, congelados, envasados y conservados de forma adecuada (Rado, 2018).

La formulación de empanizados es muy variada y conlleva la aplicación de aditivos como gomas (Berry, 1994), variación del tamaño de partículas de la mezcla (Yusof \& Lloyd, 2003), adición de carrageninas (Candogan \& Kolsarici, 2003a, 2003b) y la inclusión de almidones modificados (Sajilata \& Singhal, 2005). En especial, la inclusión de almidones modificados en la preparación de empanizados se basa en las cualidades que de termo resistencia, encapsulación del sabor, desarrollo de textura en la mezcla, así como de reducir la absorción de aceite (Abbas, K. Khalil, \& Meor Hussin, 2010). La cantidad de ingredientes que podrían contener en la mezcla, así como los procesos de cocción (temperatura, tiempo y calentamiento), podrían modificar la calidad de la corteza. Según Zhang, Yang, Ji, y Ma, (2014) últimamente han utilizado almidones como ingredientes de mejoradores de textura de la masa, reducción de contenido de aceite en alimentos fritos y rebozados.

Los almidones modificados son empleados en gran variedad de productos, estos al realizar una modificación permiten obtener características deseables (L. Plazas y J. Plazas, 2013). Así mismo, mejora la estructura crujiente de la corteza, mejorando las características de adhesión que genera entre la carne (sustrato) y la cobertura (empanizado) (Santos, Ramírez, y Solari, 2008).

\section{MATERIALES Y MÉTODOS}

\subsection{Materiales}

Para el desarrollo de la experimentación se utilizó lomo de aguja de res, pechuga de pollo y corvina rosada. Por otra parte, para la mezcla con la que se formuló el empanizado se emplearon harinas, condimentos, almidón de maíz y almidón modificado de maíz-PBP25-SF6450 y CMC de mercados locales del cantón Latacunga. Para el proceso de fritura se empleó aceite comercial, cuyos ingredientes son: aceite puro de soya, oleína de palma, ácido cítrico y antioxidantes BHT.

- Preparación de la mezcla de revestimiento

Los ingredientes fueron pesados en base a las formulaciones presentadas en la Tabla 1 .
Tabla 1. Formulación de la mezcla empanizadora

\begin{tabular}{lccc}
\hline & T1 & T2 & T3 \\
& $(\%)$ & $(\%)$ & $(\%)$ \\
\hline Almidón modificado de & 10 & 20 & 30 \\
maíz & & & \\
Almidón de maíz & 35 & 25 & 15 \\
Harina de maíz & 20 & 20 & 20 \\
Harina de arroz & 25 & 25 & 25 \\
Harina de trigo fortificada & 10 & 10 & 10 \\
\hline Carboximetil celulosa & 1 & 1 & 1 \\
Condimentos & 10,5 & 10,5 & 10,5 \\
Sal & 4 & 4 & 4 \\
\hline Fórmulas aplicadas para la evaluación de empanizados
\end{tabular}

En la Tabla 1, se presentan las formulaciones aplicadas en el presente estudio. En cada formulación se resalta que existe una variación de almidón modificado - AM entre el 10\% y el $30 \%$ para cada tratamiento. Según el incremento de AM, disminuye la cantidad de almidón de maíz de cada formulación.

Para establecer el nivel de eficiencia de la aplicación de cada tratamiento, el estudio considera la incorporación de testigo, a partir de un empanizado de marca comercial, el cual no contiene almidón modificado en su composición. El proceso de aplicación de esta marca comercial - MC siguió el mismo proceso aplicado para cada tratamiento, así como fue sometida a los análisis sometidos a cada tratamiento.

2.2. Desarrollo del proceso de empanizados de carne pollo, pescado y res

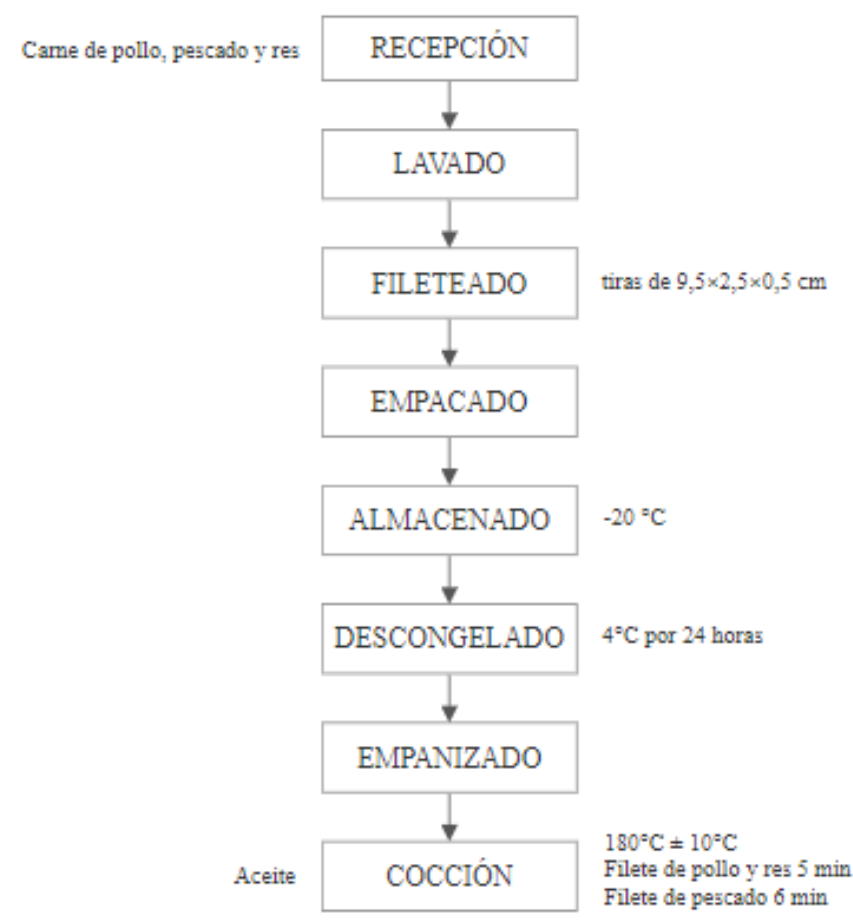

Figura 1. Diagrama de flujo para la preparación de las muestras de empanizado en fritura

En la Figura 1, se presenta el proceso de recepción, aplicación del empanizado y cocción en aceite de cada tratamiento aplicado con la variación de la formulación de empanizado. En este proceso se mantienen constantes el tamaño de los trozos de carne, la temperatura de almacenamiento, el periodo de descongelación, así como la temperatura del aceite de $180^{\circ} \mathrm{C}$. 


\subsection{Análisis sensorial}

Para la evaluación sensorial se aplicó una escala hedónica de cinco puntos en base a lo señalado por Paniagua (2014), para lo cual se utilizó 20 catadores semi entrenados y una hoja de cata proporcionando a cada catador cuatro muestras aleatorias y codificadas. La escala hedónica para los parámetros de olor, sabor y aceptabilidad fue 1 (muy desagradable) y 5 (muy agradable), para el color fue 1 (muy pálido) y 5 (dorado intenso) y para la textura el 1 (suave) y 5 (muy crujiente).

\subsection{Análisis instrumental de las muestras empanizadas}

- Color de revestimiento

La medición de color se realizó en la parte superficial (revestimiento) de cada una de las muestras ya sometidas a fritura mediante un colorímetro Lovibond RM-200 previamente calibrado, realizando mediciones de los siguientes parámetros $\mathrm{a}^{*}$ (verde $\{-\}$ a rojo $\{+\}$, b* (azul $\{-\}$ a amarillo $\{+\}$ y $L^{*}\left(L^{*}=0\right.$ para negro y $L^{*}=100$ para blanco), cromaticidad $\left(\mathrm{C}^{*}\right)$ y tono $\left(\mathrm{H}^{\circ}\right)$ dichos valores fueron proporcionados por el equipo. Se realizaron 10 repeticiones de cada tratamiento (Panduro, 2015).

\section{- Determinación de textura}

El análisis de perfil de textura de las muestras observadas se analizó mediante el Texturómetro de Brookfield CT3, en base a la metodología propuesta por Das, Pawar, y Modi, (2013) y Khalil (2000). Los filetes de lomo de aguja de res, pechuga de pollo y corvina rosada previamente empanizados y fritos fueron enfriados a temperatura ambiente $\left(12-14^{\circ} \mathrm{C}\right)$.

Las dimensiones de los filetes empanizados para el análisis comprendieron de $10 \mathrm{~cm}$ de largo, $3 \mathrm{~cm}$ de ancho y $1 \mathrm{~cm}$ de altura, el cual se aplicó una doble compresión hasta el 50\% de la altura original, a una velocidad de cruceta de $2 \mathrm{~mm} / \mathrm{s}$, además se empleó la sonda TA7 del elemento TA-BT-KIT. Los parámetros que se midieron fueron dureza, adhesividad, fracturabilidad, cohesividad y masticabilidad.

\section{- Determinación de humedad en corteza frita}

La determinación de humedad contempló el método oficial, AOAC (1990) mediante el cual se tomaron muestras de recubrimiento del sustrato inmediatamente después de freír cada uno de los tratamientos. Para ello se pesaron de 5 a 6 g de muestra, para posteriormente llevar a una estufa a $105^{\circ} \mathrm{C}$ hasta conseguir un peso constante. El proceso se realizó por triplicado y se calculó el porcentaje de humedad mediante la siguiente ecuación:

Donde:

$$
\text { Contenido de humedad }(\%)=\frac{p_{1}-p_{2}}{p_{m}} * 100
$$

$\mathrm{p}_{1}=$ Peso de la cápsula + muestra antes de secar en $\mathrm{g}$.

$\mathrm{p}_{2}=$ Peso de la cápsula + muestra después del secado en $\mathrm{g}$.

$\mathrm{p}_{\mathrm{m}}=$ Peso de muestra en $\mathrm{g}$.

\section{- Absorción de aceite}

El uso o absorción de aceite luego del proceso de fritura se determinó mediante lo señalado por Das, Pawar, y Modi, (2013) tomando el peso del aceite antes y después del proceso de freír. Para lo cual se realizó por triplicado, procediendo a calcular mediante la siguiente ecuación:

$$
\text { Absorción de aceite }(\%)=\frac{W_{1} \times W_{2}}{W_{3}} * 100
$$

Donde:

$\mathrm{W}_{1}=$ Peso del aceite antes de freír.

$\mathrm{W}_{2}=$ Peso del aceite después de freír.

$\mathrm{W}_{3}=$ Peso del sustrato crudo rebozado.

\subsection{Diseño experimental y análisis estadístico}

El diseño experimental aplicado fue el diseño completamente aleatorizado con un solo factor, siendo este la concentración de almidón modificado. El análisis estadístico se realizó en el programa STATGRAPHICS Centurion XVI para el análisis de varianza ANOVA de una vía. Para la comparación de los tratamientos se realizó mediante la prueba Tukey, con un nivel de significancia de $\mathrm{P} \leq 0,05$.

\section{RESULTADOS Y DISCUSIÓN}

\subsection{Análisis sensorial}

Mediante el análisis sensorial se puede dar a conocer la aceptación o rechazo de un producto, dado que permite evocar, medir, analizar, e interpretar reacciones de las características de los alimentos lo cual son percibidos por los sentidos (Severiano, 2010).

Tabla 2. Resultados promedio del análisis sensorial

\begin{tabular}{cccc}
\hline \multirow{2}{*}{ Tratamiento } & Tipo de & \multicolumn{2}{c}{ Atributos sensoriales } \\
\cline { 3 - 4 } & empanizado & Textura & Aceptabilidad \\
\hline \multirow{2}{*}{ M.C } & Pollo & $4,10 \pm 0,91$ & $4,20 \pm 0,70$ \\
& Pescado & $4,30 \pm 0,80$ & $3,90 \pm 0,91$ \\
& Res & $4,40 \pm 0,50$ & $4,10 \pm 0,64$ \\
\hline \multirow{2}{*}{ T1 } & Pollo & $3,35 \pm 0,81$ & $3,45 \pm 0,76$ \\
& Pescado & $3,65 \pm 0,81$ & $3,60 \pm 0,60$ \\
& Res & $3,75 \pm 0,91$ & $3,50 \pm 0,83$ \\
\hline \multirow{2}{*}{ T2 } & Pollo & $3,60 \pm 0,82$ & $3,55 \pm 0,69$ \\
& Pescado & $3,50 \pm 1,10$ & $3,55 \pm 0,69$ \\
& Res & $3,40 \pm 0,68$ & $3,50 \pm 0,69$ \\
\hline \multirow{2}{*}{ T3 } & Pollo & $3,80 \pm 0,77$ & $4,00 \pm 0,86$ \\
& Pescado & $3,95 \pm 0,94$ & $3,75 \pm 1,12$ \\
& Res & $4,10 \pm 0,91$ & $3,65 \pm 0,59$ \\
\hline
\end{tabular}

$\bar{x} \pm$ desviación respecto a 20 catadores. M.C (marca comercial), T1 (10\% de almidón modificado), T2 (20\% de almidón modificado), T3 (30\% de almidón modificado).

Por una parte, en la Tabla 2, se presentan los resultados del análisis sensorial, para los parámetros de Textura y Aceptabilidad. En esta tabla se observa que existe una relación significativa de las muestras de T2, en relación con MC y T1 y T3. De acuerdo al análisis efectuado, se puede considerar comparativamente que aquella mezcla compuesta con un $20 \%$ 
de almidón modificado posee mejores características, según la evaluación sensorial.

Por otra parte, en las Figuras 2, 3 y 4 los resultados evidencian una variación de color comprendida entre una escala hedónica cercano al "dorado" y "dorado intenso". En cuanto al olor y sabor existe una mayor puntuación para los empanizados con harina comercial con una escala hedónica de "agradable". Mientras que para el atributo textura existe una preferencia en el T3 con una escala hedónica de "crujiente" seguido de los empanizados con harina comercial, mejorando este atributo al incrementar su concentración.

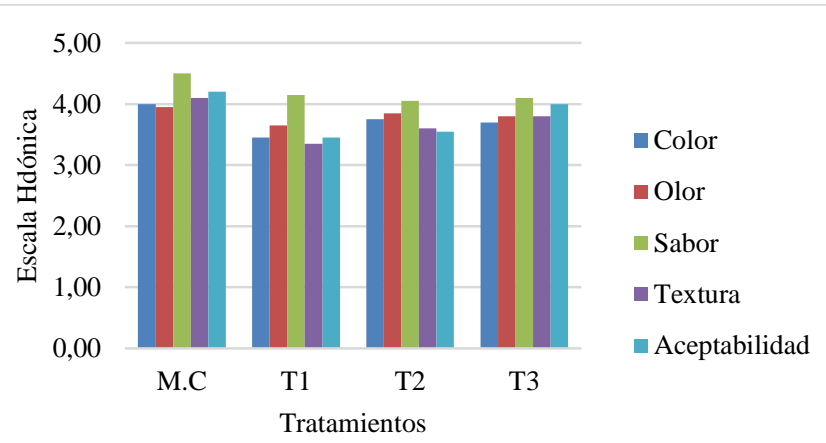

Figura 2. Valoración hedónica de los empanizados con filetes de pechuga de pollo a diferentes concentraciones de almidón modificado. $\mathrm{MC}=$ marca comercial, $\mathrm{T}=$ Tratamiento.

En el análisis de Nuggets de pollo frito su textura varió de 3,5 a 4,5 en su escala hedónica al adicionar $10 \%$ de almidón modificado de maíz con alto contenido de amilosa, siendo este aceptable por los panelistas (Vongsawasdi, 2014). Finalmente se puede observar que el mejor tratamiento corresponde al T3 para los tres tipos de carne, lo cual evidencia que la variación de concentraciones interfiere en el mejoramiento de sus atributos, logrando alcanzar una mezcla en la cual genere aceptación entre los tratamientos MC y T3.

Según Agromeat (2011), la utilización de almidones modificados principalmente son empleados en productos cárnicos primordialmente de pollo a fin de incrementar altos rendimientos, mejoramiento en la textura y la palatabilidad del producto.

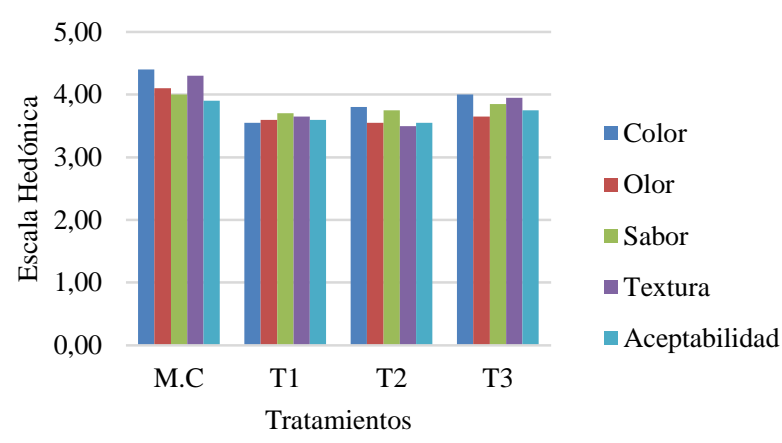

Figura 3. Valoración hedónica de los empanizados con filetes de corvina rosada a diferentes concentraciones de almidón modificado.

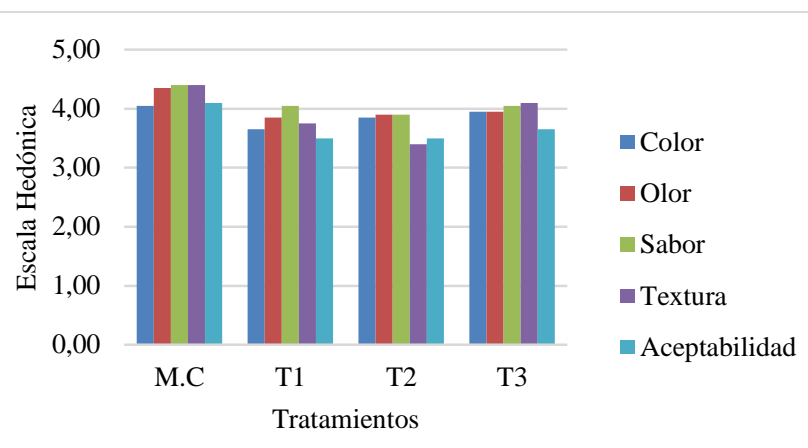

Figura 4. Valoración hedónica de los empanizados con filetes de lomo de aguja de res a diferentes concentraciones de almidón modificado.

\subsection{Análisis instrumental de los empanizados}

\section{- Color}

En las Tablas 3, 4 y 5 se muestran los resultados de los parámetros de color $\left(\mathrm{L}^{*}, \mathrm{a},{ }^{*}, \mathrm{~b}^{*}, \mathrm{C}^{*} \mathrm{y} \mathrm{H}^{\circ}\right)$ de las muestras empanizadas de pollo, pescado y res respectivamente. Los resultados muestran que los tratamientos formulados presentaron coloraciones más claras; es decir con mayor luminosidad, dando como resultado una coloración menos dorada. En cuanto al parámetro a* se evidencian valores menores en las muestras con carne de res, lo cual podría ser una producción menor de la reacción de Maillard (Bengtson, Cundiff, y Flick, 2006). Con respecto a cromaticidad (C*) y tono $\left(\mathrm{H}^{\circ}\right)$ presentan resultados similares con estudios realizados por Albert, Salvador, Hough, y Fiszman, (2014), quienes señalan que las cortezas fritas de Nuggets obtuvieron valores de 32,5 y 67,5 respectivamente, resultando un color más amarillo y menos saturado, generando una coloración marrón dorado en la evaluación sensorial.

Tabla 3. Parámetros de color: $\mathrm{L}^{*}, \mathrm{a}^{*}, \mathrm{~b}^{*}, \mathrm{C}^{*}, \mathrm{H}^{\circ}$ de las muestras empanizados con pollo.

\begin{tabular}{cccccc}
\hline T & $\mathrm{L}^{*}$ & $\mathrm{a}^{*}$ & $\mathrm{~b}^{*}$ & $\mathrm{C}^{*}$ & $\mathrm{H}^{\circ}$ \\
\hline \multirow{2}{*}{ M.C } & 46,07 & 16,16 & 32,8 & 36,59 & 63,76 \\
& $\pm 2,97^{\mathrm{a}}$ & $\pm 1,07^{\mathrm{a}}$ & $\pm 1,51^{\mathrm{a}}$ & $\pm 1,35^{\mathrm{a}}$ & $\pm 1,97^{\mathrm{a}}$ \\
$\mathrm{T} 1$ & 52,32 & 13,57 & 28,48 & 31,63 & 64,28 \\
& $\pm 3,50^{\mathrm{c}}$ & $\pm 1,65^{\mathrm{b}}$ & $\pm 3,79^{\mathrm{bc}}$ & $\pm 3,38^{\mathrm{bc}}$ & $\pm 4,17^{\mathrm{a}}$ \\
$\mathrm{T} 2$ & 48,52 & 11,72 & 26,56 & 29,01 & 66,46 \\
& $\pm 2,41^{\mathrm{ab}}$ & $\pm 1,76^{\mathrm{c}}$ & $\pm 1,55^{\mathrm{c}}$ & $\pm 1,88^{\mathrm{c}}$ & $\pm 3,11^{\mathrm{a}}$ \\
T3 & 50,57 & 15,29 & 30,20 & 33,88 & 63,05 \\
& $\pm 2,39^{\mathrm{bc}}$ & $\pm 1,29^{\mathrm{a}} \mathrm{b}$ & $\pm 3,05^{\mathrm{ab}}$ & $\pm 2,92^{\mathrm{ab}}$ & $\pm 2,64^{\mathrm{a}}$ \\
\hline Tratamientos & T T, Luminosidad &
\end{tabular}

Tratamientos $=\mathrm{T}$, Luminosidad $=\mathrm{L}^{*},+$ rojo $=\mathrm{a}^{*}$, Cromaticidad $=\mathrm{C}^{*}$, Ángulo hue $=\mathrm{H}^{\circ}$

Tabla 4. Parámetros de color: $\mathrm{L}^{*}, \mathrm{a}^{*}, \mathrm{~b}^{*}, \mathrm{C}^{*}, \mathrm{H}^{\circ}$ de las muestras empanizados con pescado.

\begin{tabular}{llllll}
\hline T & L $^{*}$ & $\mathrm{a}^{*}$ & $\mathrm{~b}^{*}$ & $\mathrm{C}^{*}$ & $\mathrm{H}^{\circ}$ \\
\hline \multirow{2}{*}{ M.C } & 37,25 & 13,63 & 21,32 & 25,31 & 57,39 \\
& $\pm 3,43^{\mathrm{a}}$ & $\pm 1,53^{\mathrm{a}}$ & $\pm 2,68^{\mathrm{b}}$ & $\pm 2,85^{\mathrm{a}}$ & $\pm 2,52^{\mathrm{a}}$ \\
& 44,13 & 11,20 & 24,71 & 27,15 & 65,36 \\
$\mathrm{~T} 1$ & $\pm 3,63^{\mathrm{b}}$ & $\pm 0,96^{\mathrm{b}}$ & $\pm 3,45^{\mathrm{a}}$ & $\pm 3,35^{\mathrm{a}}$ & $\pm 2,74^{\mathrm{b}}$ \\
& 40,50 & 11,02 & 22,58 & 25,16 & 64,10 \\
$\mathrm{~T} 2$ & $\pm 3,20^{\mathrm{ab}}$ & $\pm 1,68^{\mathrm{b}}$ & $\pm 1,55^{\mathrm{ab}}$ & $\pm 1,97^{\mathrm{a}}$ & $\pm 2,71^{\mathrm{b}}$ \\
& 40,22 & 11,26 & 23,51 & 26,11 & 64,33 \\
T3 & $\pm 3,23^{\mathrm{ab}}$ & $\pm 1,14^{\mathrm{b}}$ & $\pm 2,27^{\mathrm{ab}}$ & $\pm 2,12^{\mathrm{a}}$ & $\pm 3,05^{\mathrm{b}}$
\end{tabular}

M.C (marca comercial), T1 (10\% de almidón modificado), T2 (20\% de almidón modificado), T3 (30\% de almidón modificado). Los superíndices diferentes indican diferencias significativas, al 95\% de confianza evaluada con una prueba Tukey. 
Tabla 5. Parámetros de color: $\mathrm{L}^{*}, \mathrm{a}^{*}, \mathrm{~b}^{*}, \mathrm{C}^{*}, \mathrm{H}^{\circ}$ de las muestras empanizados con res.

\begin{tabular}{llllll}
\hline T & $\mathrm{L}^{*}$ & $\mathrm{a}^{*}$ & $\mathrm{~b}^{*}$ & $\mathrm{C}^{*}$ & $\mathrm{H}^{\circ}$ \\
\hline \multirow{2}{*}{$\mathrm{M} . \mathrm{C}$} & 37,25 & 13,63 & 21,32 & 25,31 & 57,39 \\
& $\pm 3,43^{\mathrm{a}}$ & $\pm 1,53^{\mathrm{a}}$ & $\pm 2,68^{\mathrm{b}}$ & $\pm 2,85^{\mathrm{a}}$ & $\pm 2,52^{\mathrm{a}}$ \\
$\mathrm{T} 1$ & 44,13 & 11,20 & 24,71 & 27,15 & 65,36 \\
& $\pm 3,63^{\mathrm{b}}$ & $\pm 0,96^{\mathrm{b}}$ & $\pm 3,45^{\mathrm{a}}$ & $\pm 3,35^{\mathrm{a}}$ & $\pm 2,74^{\mathrm{b}}$ \\
$\mathrm{T} 2$ & 40,50 & 11,02 & 22,58 & 25,16 & 64,10 \\
& $\pm 3,20^{\mathrm{ab}}$ & $\pm 1,68^{\mathrm{b}}$ & $\pm 1,55^{\mathrm{ab}}$ & $\pm 1,97^{\mathrm{a}}$ & $\pm 2,71^{\mathrm{b}}$ \\
$\mathrm{T} 3$ & 40,22 & 11,26 & 23,51 & 26,11 & 64,33 \\
& $\pm 3,23^{\mathrm{ab}}$ & $\pm 1,14^{\mathrm{b}}$ & $\pm 2,27^{\mathrm{ab}}$ & $\pm 2,12^{\mathrm{a}}$ & $\pm 3,05^{\mathrm{b}}$ \\
\hline
\end{tabular}

M.C (marca comercial), T1 (10\% de almidón modificado), T2 (20\% de almidón modificado), T3 (30\% de almidón modificado). Los superíndices diferentes indican diferencias significativas, al 95\% de confianza evaluada con una prueba Tukey.

\subsection{Análisis de perfil de textura}

Los valores de perfil de textura para los empanizados se muestran en la Tabla 6. La dureza en el T3 alcanza un valor similar al tratamiento con harina comercial; mientras que el T1 tiene un valor muy elevado. Según Prestes et al., (2014) al presentar una mayor dureza, presentan una menor humedad en el producto por ende requiere de más energía para la masticación.

Los tres tipos de carne plantean que en el $\mathrm{T} 1$ se requiere de más energía y fuerza para la masticación, lo cual podría ser efecto de una carne con mayor cantidad de pérdida de agua. Inclusive, sensorialmente este tratamiento no fue muy aceptable para los catadores. Ziobro, Korus, Witczak, y Juszczak, (2012); Barbut, (2018) señalan que el reemplazo parcial de almidón nativo por almidón modificado influye principalmente en la disminución de la dureza, masticabilidad y gomosidad. Es decir, esto se genera por interacciones de las proteínas de la carne juntamente con el almidón modificado, generando al producto más digerible y aceptable. En cuanto al T3 se podría señalar que existe un equilibro, dado que se asemeja a la crujencia obtenida en las muestras con harina

Determinación de humedad de la corteza y absorción de aceite Las muestras con adición de almidón modificado obtuvieron una mayor humedad en la corteza, lo cual podría ser efecto de una absorción menor de aceite en comparación con las cortezas de la harina comercial (Tabla 8).

Tabla 6. Humedad en la corteza de los empanizados TIPO DE CORTEZA DE EMPANIZADO

\begin{tabular}{lccc}
\hline & Pollo & Pescado & Res \\
\hline M.C & $8,32 \pm 0,92^{\mathrm{a}}$ & $13,31 \pm 7,51^{\mathrm{a}}$ & $8,30 \pm 2,84^{\mathrm{a}}$ \\
T1 & $7,97 \pm 0,29^{\mathrm{a}}$ & $18,76 \pm 4,40^{\mathrm{a}}$ & $7,95 \pm 2,27^{\mathrm{a}}$ \\
T2 & $9,63 \pm 2,61^{\mathrm{a}}$ & $19,38 \pm 3,37^{\mathrm{a}}$ & $7,49 \pm 3,98^{\mathrm{a}}$ \\
T3 & $10,55 \pm 3,03^{\mathrm{a}}$ & $16,87 \pm 1,16^{\mathrm{a}}$ & $8,34 \pm 1,57^{\mathrm{a}}$ \\
\hline
\end{tabular}
M.C (marca comercial), T1 (10\% de almidón modificado), T2 (20\% de almidón modificado), T3 (30\% de almidón modificado). Los superíndices diferentes indican diferencias significativas, al 95\% de confianza evaluada con una prueba Tukey.

Los almidones modificados han sido utilizados para disminuir el contenido de aceite de los alimentos fritos y rebozados, debido al alto contenido de amilosa de los gránulos de almidón, proporcionando una mejor barrera dando como efecto que el aceite no penetre al sustrato; el contenido de aceite es importante ya que disminuye la vida útil a través del proceso de oxidación (Zhang et al., 2014).

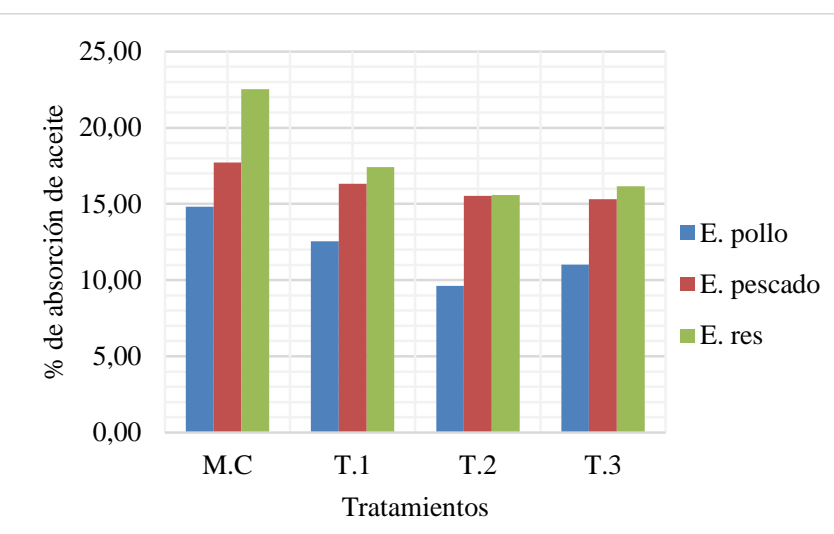

Figura 5. Porcentaje de absorción de aceite en diferentes tipos de carne y concentración de almidón modificado

La utilización de almidones modificados tiene la ventaja de formar una película perfecta para el sustrato, evitando así una pérdida de humedad y la reducción de absorción de aceite. Además, puede usarse para mejorar aún más la adhesión en batters y al desarrollo de una textura crujiente en productos rebozados o empanizados.

Tabla 7. Porcentaje de absorción de aceite

\begin{tabular}{cccc}
\hline \multirow{2}{*}{ Tratamiento } & \multicolumn{3}{c}{ Tipo de empanizado } \\
\cline { 2 - 4 } & Pollo & Pescado & Res \\
\hline M.C & $14,83 \pm 0,50^{\mathrm{b}}$ & $17,72 \pm 6,85^{\mathrm{a}}$ & $22,54 \pm 2,63^{\mathrm{b}}$ \\
T.1 & $12,55 \pm 0,26^{\mathrm{ab}}$ & $16,32 \pm 5,17^{\mathrm{a}}$ & $17,42 \pm 0,47^{\mathrm{ab}}$ \\
T.2 & $9,63 \pm 1,68^{\mathrm{a}}$ & $15,53 \pm 1,14^{\mathrm{a}}$ & $15,58 \pm 3,23^{\mathrm{a}}$ \\
T.3 & $11,01 \pm 2,66^{\mathrm{ab}}$ & $15,32 \pm 1,40^{\mathrm{a}}$ & $16,16 \pm 1,58^{\mathrm{a}}$
\end{tabular}

M.C (marca comercial), T1 (10\% de almidón modificado), T2 (20\% de almidón modificado), T3 (30\% de almidón modificado). Los superíndices diferentes indican diferencias significativas, al 95\% de confianza evaluada con una prueba Tukey.

En la Tabla 7 se presentan los resultados, donde las muestras con harina comercial tuvieron mayor cantidad de absorción de aceite a diferencia de entre las muestras con almidón modificado de maíz. Las muestras con M.C obtuvieron un $14,83 \%, 17,72 \%$ y $22,54 \%$ para empanizados con carne de pollo, pescado y res respectivamente, mientras que el T3 con un valor de $11,01 \%$ para pollo, $15,32 \%$ en pescado y $16,16 \%$ res empanizados. Es decir, en relación con MC, para las tres carnes, T2 presenta una menor absorción de aceite, con una diferencia para T3 en el caso de las muestras evaluadas de pescado.

\section{CONCLUSIONES}

Este estudio tiene como objetivo analizar la adición de almidón modificado para la elaboración de empanizado, evaluado sobre tres muestras de carne (res, pescado, pollo). Los resultados demuestran que la inclusión de almidón modificado puede ser utilizada en una mezcla empanizadora, dado por las características obtenidas tras el proceso de fritura. Mediante la evaluación sensorial el tratamiento con 30\% de almidón modificado presentó una mejor aceptación sensorial, seguido de las muestras con harina comercial, evidenciando 
principalmente en la textura un mejoramiento al incrementar su concentración, obteniendo así una corteza crujiente.

Las propiedades de color mostraron cierta variación en un rango mínimo, dando valores altos en las coordenadas $\mathrm{a}^{*} \mathrm{y} \mathrm{b}^{*}$ con un $\mathrm{H}^{\circ}$ comprendidas entre $66,46 \pm 3,11$ a $59,65 \pm 1,83$ con una tendencia hacia el color dorado característico de los alimentos fritos. En cuanto a los parámetros de textura, estos revelaron que la cantidad de almidón mejora sus características, dado a que requiere de una fuerza y energía menor para la masticación a diferencia de la muestra con mayor cantidad de almidón nativo, lo cual podría ser por efecto de una mayor pérdida de agua y la formación de una corteza más dura, limitando así la utilización del almidón nativo en procesos de temperaturas altas. El porcentaje de humedad en la corteza no presentó alguna diferencia significativa en relación con la cantidad de almidón. Sin embargo redujo el porcentaje de absorción de aceite en los empanizados, lo cual podría ser efecto por la cantidad de amilosa que puede contener el almidón modificado creando una película que impide el ingreso del aceite.

\section{REFERENCIAS}

Abbas, K. A., K. Khalil, S., \& Meor Hussin, A. S. (2010). Modified Starches and Their Usages in Selected Food Products: A Review Study. Journal of Agricultural Science, 2(2). https://doi.org/10.5539/jas.v2n2p90

Agromeat. (2011). Utilizando almidones para maximizar rendimientos de procesamiento. https://www.agromeat.com/41485/utilizandoalmidones-para-maximizar-rendimientos-deprocesamiento

Albert, A., Salvador, A., Hough, G., \& Fiszman, S. (2014). Influence of outer layer formulation on the sensory properties of microwaved breaded nuggets. International Journal of Food Properties, 17(4), 829841. https://doi.org/10.1080/10942912.2011.604892

AOAC. Official Methods of Analysis of the Association of Official Analytical Chemists, 35 Choice Reviews Online $\S$ (1990). https://doi.org/10.5860/choice.35-0912

Barbut, S. (2018). Effects of regular and modified potato and corn starches on frankfurter type products prepared with vegetable oil. Italian Journal of Food Science, 30(4), 801-808. https://doi.org/10.14674/IJFS-1084

Bengtson, R., Cundiff, J. S., \& Flick, G. J. (2006). The Effect of Novel Frying Methods on Quality of Breaded Fried Foods Master of Science The Effect of Novel Frying Methods on Quality of Breaded Fried Foods. Systems Engineering.

Berry, B. W. (1994). Properties of Low-Fat, Nonbreaded Pork Nuggets with Added Gums and Modified Starches. Journal of Food Science, 59(4), 742-746. https://doi.org/10.1111/j.1365-2621.1994.tb08117.x
Candogan, K., \& Kolsarici, N. (2003a). Storage stability of low-fat beef frankfurters formulated with carrageenan or carrageenan with pectin. Meat Science, 64(2), 207-214. https://doi.org/10.1016/S0309-1740(02)00182-1

Candogan, K., \& Kolsarici, N. (2003b). The effects of carrageenan and pectin on some quality characteristics of low-fat beef frankfurters. Meat Science, 64(2), 199206. https://doi.org/10.1016/S0309-1740(02)00181-X

Das, R., Pawar, D. P., \& Modi, V. K. (2013). Quality characteristics of battered and fried chicken: comparison of pressure frying and conventional frying, 50(April), 284-292. https://doi.org/10.1007/s13197011-0350-z

Dávalos, L. (2016). Desarrollo de nuggets de bonito (Sarda chiliensis chiliensis) bajos en calorías y con la adición de chía (Salvia hispánica) como antioxidante. (Tesis pregrado). Universidad Nacional de San Agustin, Arequipa, Perú.

Khalil, A. H. (2000). Quality characteristics of low-fat beef patties formulated with modified corn starch and water. Food Chemistry. 68. DOI: 10.1016/S03088146(99)00156-9

Montes, N., Millar, I., \& Provoste, R. (2016). Absorción de aceite en alimentos fritos. Revista chilena de nutrición, 43(1), 87-91. https://dx.doi.org/10.4067/S071775182016000100013

Panduro, C. (2015). Efecto de la sustitución de harina de trigo por harina de quinua (Chenopodium quinoa) sobre el contenido de proteína, color, firmeza y aceptabilidad general de nuggets de pollo. (Tesis pregrado). Universidad Privada Antenor Orrego, Trujillo, Perú.

Paniagua, N. (2014). Aplicación de harinas extrusionadas en la mejora de la calidad de rebozados. (Trabajo maestría). Universidad de Valladolid, Palencia, España.

Plazas, L., \& Plazas, J. (2013). Aplicación de minería de datos para la segmentación de clientes que comoran materias primas derivadas del maíz para la generación de estrategias de cominucación. (Tesis pregrado). Universidad Piloto de Colombia, Bogotá, Colombia.

Prestes, R. C., Silva, L. B., Torri, A. M. P., Kubota, E. H., Rosa, C. S., Roman, S. S., ... Demiate, I. M. (2014). Sensory and physicochemical evaluation of low-fat chicken mortadella with added native and modified starches. Journal of Food Science and Technology, 52(7), 4360-4368. https://doi.org/10.1007/s13197-0141496-2

Rado, R. (2018). Empanizados de filetes congelados de trucha arco iris (Oncorhynchus mykiss) sazonados y enriquecidos con quinua (Chenopodium quinoa). (Tesis pregrado). Universidad Nacional de San Agustín De Arequipa, Arequipa, Perú. 
Sahin, S., \& Gülün, S. (2009). Advances in deep-fat frying of foods. Boca Raton: CRC Press.

Sajilata, M. G., \& Singhal, R. S. (2005). Specialty starches for snack foods. Carbohydrate Polymers, 59(2), 131-151. https://doi.org/10.1016/j.carbpol.2004.08.012

Santos, R. M., Ramírez, A., \& Solari, A. (2008). Desarrollo de formulaciones de batido como alternativas a productos comerciales utilizados en el empanizado de porciones de calamar gigante. Inst. tecnol. pesq. 8, 57-64.

Severiano, P. (2010). Manual de evaluación sensorial.

Vongsawasdi, P. (2014). Using modified starch to decrease the oil absorption in fried battered chicken, (January 2008).

Yusof, M., \& Lloyd, W. (2003). Efect of Breading Particle Size on Coating Adhesion in Breaded, Fried Chicken Breasts. Coating, 27(Scott 1987), 103-113.

Zhang, L., Yang, M., Ji, H., \& Ma, H. (2014). Some physicochemical properties of starches and their influence on color, texture, and oil content in crusts using a deep-fat-fried model. CYTA - Journal of Food, 12(4), $347-354$ https://doi.org/10.1080/19476337.2014.887148

Ziobro, R., Korus, J., Witczak, M., \& Juszczak, L. (2012). Influence of modified starches on properties of glutenfree dough and bread. Part II: Quality and staling of gluten-free bread. Food Hydrocolloids, 29(1), 68-74. https://doi.org/10.1016/j.foodhyd.2012.02.009

\section{BIOGRAFÍAS}

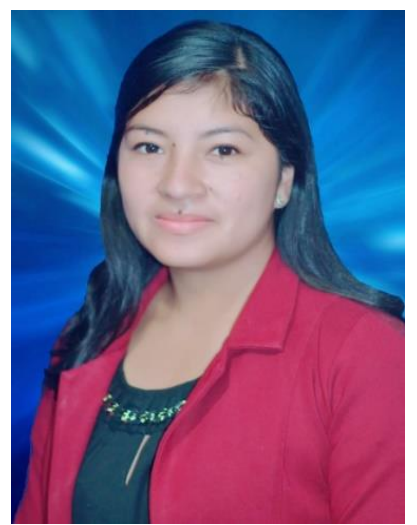

Norma Alexandra Bautista Muso, nació el 15 de Agosto de 1994, en la provincia de Cotopaxi cantón Latacunga. En el año 2012, obtuvo el título de Bachiller Técnico Agropecuario: Especialización Explotaciones Agropecuarias otorgado por el Colegio "Simón Rodríguez". En el año 2019, inició el proyecto de investigación bajo el tema "Evaluación de una mezcla empanizadora, con inclusión de almidón modificado, para su aplicación en carnes", para la obtención del título de Ingeniera en Alimentos en la Universidad Técnica de Ambato. Actualmente, encamina en la creación de su propio emprendimiento en la industria láctea. ORCID: https://orcid.org/0000-0002-2683-5730

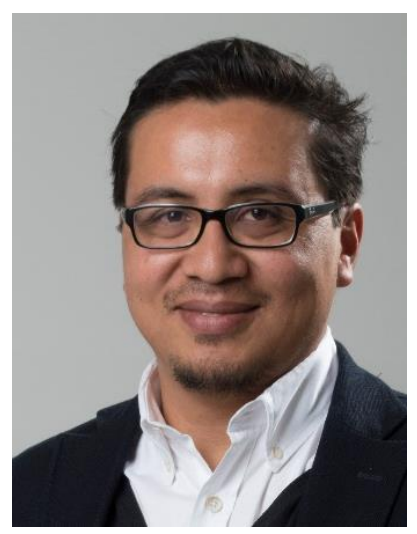

Christian Franco Crespo, Doctor en Economía Agraria, por la Universidad Politécnica de Madrid. Quiteño y actualmente cumple funciones como Docente Investigador en la Universidad Técnica de Ambato. Se ha desempeñado como coordinador de proyectos de investigación, conferencista y editor de revistas científicas. ORCID: http://orcid.org/00000002-4818-4350 
